
GUANAJUATO.

\title{
EVALUACIÓN DE SERVICIO EN LAS EMPRESAS DE LA RUTA DEL VINO EN EL ESTADO DE GUANAJUATO
}

\section{SERVICE EVALUATION IN THE COMPANIES OF THE WINE ROUTE IN THE STATE OF GUANAJUATO}

\author{
Adriana López Barberena*, Laura Elena Zárate Negrete**,

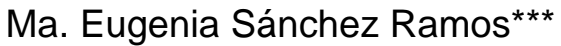

\begin{abstract}
*Maestra en Comercio Electrónico y Maestra en Mercadotecnia. Universidad Tecnológica de León. ORCID: http://orcid.org/0000-0002-0861-9807.

**Doctora en Desarrollo Humano. Universidad de Guanajuato. Email: lezarate@ugto.mx. ORCID: http://orcid.org/0000-0001-7520-8314.
\end{abstract}

***Doctora en Arquitectura, Diseño y Urbanismo. Universidad de Guanajuato. Email: sanchez.me@ugto.mx. ORCID: http://orcid.org/0000-0002-0861-9807.

Dirección para recibir correspondencia: abarberena@utleon.edu.mx

Fecha de recibido: 25 de agosto de 2019

DOI: https://doi.org/10.19136/hitos.a25n73.3568

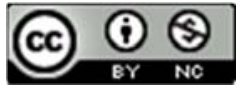


EVALUACIÓN DE SERVICIO EN LAS EMPRESAS DE LA RUTA DEL VINO EN EL ESTADO DE GUANAJUATO.

\title{
RESUMEN
}

OBJETIVO: Determinar las prácticas comerciales en el servicio al cliente para mejorar las estrategias mercadológicas de la zona. Las empresas elegidas son productoras y comercializadoras conocidas como "viñedos boutique".

MATERIAL Y MÉTODO: La investigación es explicativa, descriptiva, exploratoria de corte transversal y enfoque mixto. El instrumento de medición corresponde a una guía de observación indirecta no participante para evaluar dimensiones del pentágono de Babson College utilizando la escala de Likert.

RESULTADOS: Entre los hallazgos se identificó que los viñedos son competitivos en la parte de la infraestructura y el servicio, pero se tienen carencias importantes en el área de los productos y su comercialización.

CONCLUSIONES: El impacto de esta evaluación es alto porque se carece de información en el ámbito del servicio en la ruta vitivinícola en el estado de Guanajuato.

PALABRAS CLAVE: Servicio al cliente. Evaluación de estrategias. Viñedos. Ruta del Vino.

\begin{abstract}
OBJECTIVE: To determine the commercial practices in the customer service to improve the marketing strategies of the area. The chosen companies are producers and marketers known as "boutique vineyards".

MATERIAL AND METHOD: The research is explanatory, descriptive, cross-sectional exploratory and mixed approach. The measuring instrument corresponds to a non-participant indirect observation guide to evaluate dimensions of the Babson College pentagon using the Likert scale.
\end{abstract}


EVALUACIÓN DE SERVICIO EN LAS EMPRESAS DE LA RUTA DEL VINO EN EL ESTADO DE GUANAJUATO.

RESULTS: Among the findings it was identified that the vineyards are competitive in the part of the infrastructure and the service but there are important shortcomings in the area of the products and their commercialization.

CONCLUSIONS: The impact of this evaluation is high because there is a lack of information in the field of service on the wine route in the state of Guanajuato.

KEYWORDS: Customer Service. Strategy evaluation. Vineyards. Wine Route.

\section{INTRODUCCIÓN}

Actualmente, la medición del servicio es uno de los elementos clave en la organización, de este tipo de medición se desprenden acciones concretas para mejorar las estrategias de comercialización del mismo. Uno de los aspectos más importantes es, empezar a determinar si el servicio en el punto de venta es lo mismo de lo que se ofrece en la publicidad o en las recomendaciones de los usuarios; por lo tanto, las experiencias se deben medir para encontrar indicadores que nos den puntos de fortaleza y puntos de mejora.

El objetivo de la presente investigación es, conocer los aspectos a mejorar en el momento de ofertar servicios especializados en el sector vitivinícola en el estado de Guanajuato, además la importancia de estas empresas radica en los ingresos generados por este sector, que ya son considerables; por ello, los viñedos elegidos son líderes en el sector en la ruta del vino del estado. De la misma manera, las empresas son conocidas internacionalmente por sus productos, como el caso del viñedo ubicado en el municipio de Dolores Hidalgo, en el estado de Guanajuato, que cuenta con premios como "el Premio Guanajuato a la Competitividad Turística como Mejor Producto Turístico 2014". Asimismo, se trabajó con el viñedo ubicado en la comunidad de Sangre de Cristo, en el municipio de Guanajuato, en el estado de Guanajuato, mismo que ha recibido varios reconocimientos al albergar diferentes eventos con empresas internacionales, como lo son: eventos para las armadoras de automóviles de la zona industrial del municipio de Silao. 
EVALUACIÓN DE SERVICIO EN LAS EMPRESAS DE LA RUTA DEL VINO EN EL ESTADO DE GUANAJUATO.

Según las Estadísticas del Banco Mundial el impacto de los servicios en el Producto Interno Bruto (PIB) ha crecido de manera importante en los últimos años. En las zonas como América del Norte y la comunidad Económico Europea, se presentan datos interesantes que reflejan el alza de este sector. A continuación, se presentan las dos zonas representativas: América del Norte y la Comunidad Económica Europea (ver tabla 1).

Tabla 1

Impacto de los servicios en el PIB

\begin{tabular}{lcccccccc}
\hline País & 2010 & 2011 & 2012 & 2013 & 2014 & 2015 & 2016 & 2017 \\
\hline México & 4.00507 & 4.00146 & 3.9706 & 3.94241 & 4.23276 & 4.745083 & 5.321061 & 5.656759 \\
Estados Unidos & 6.49588 & 6.849 & 6.8568 & 6.95904 & 7.01101 & 6.883161 & 6.812165 & 6.911347 \\
Canadá & 10.8684 & 10.7729 & 11.03 & 11.0359 & 11.1048 & 11.72339 & 11.85559 & 11.71464 \\
Unión Europea & 18.8593 & 19.4998 & 20.469 & 21.2371 & 22.1267 & 23.66879 & 23.85551 & 24.48724 \\
\hline
\end{tabular}

Nota: Cifras en miles de millones de dólares.

Fuente: Elaboración propia con información del Informe del Banco Mundial (2017).

Como se observa en la tabla 1, en el caso de México se ve que en los últimos años se tiene un crecimiento en el impacto del PIB a través de los servicios desde el 2014 en adelante. Entre los datos obtenidos, es interesante analizar que hubo un retroceso del 2011 al 2013, pero a partir del 2014 mejoró de manera constante. En el caso de los Estados Unidos, las cifras de los años 2015, 2016 y 2017, son menores que las que se registraron en 2014, lo cual es un decrecimiento en los servicios, situación que vale la pena analizar en posteriores estudios. Además, Canadá es el país que tiene el mayor impacto en el PIB, por el comercio de servicios de los tres países que pertenecen a la zona de Norteamérica. Evidentemente, la Comunidad Económica Europea genera datos de impacto positivo en su PIB, que son el doble en comparación con Canadá y casi el triple en comparación con Estados Unidos y México. Todo esto demuestra la importancia actual de los servicios en diferentes zonas económicas del mundo.

Asimismo, existen datos publicados por el Banco Mundial que relacionan la importancia de las actividades económicas de productos y servicios y su impacto en el PIB, específicamente en México, esto indica el incalculable valor de los servicios como parte de la economía de este país (ver tabla 2). 
EVALUACIÓN DE SERVICIO EN LAS EMPRESAS DE LA RUTA DEL VINO EN EL ESTADO DE GUANAJUATO.

Tabla 2

Impacto en el PIB de los servicios en la economía mexicana

\begin{tabular}{lllll}
\hline & 1990 & 2000 & 2010 & 2017 \\
\hline Exportaciones de bienes y servicios & $19 \%$ & $25 \%$ & $30 \%$ & $38 \%$ \\
Importaciones de bienes y servicios & $20 \%$ & $27 \%$ & $31 \%$ & $40 \%$ \\
\hline
\end{tabular}

Nota: \% de incremento en el impacto al PIB.

Fuente: Elaboración propia con datos del reporte de México del Banco Mundial (2017).

Según datos presentados en la tabla 2, existe una evolución favorable en los años 1990, 2000, 2010 y 2017, en el crecimiento del impacto de los servicios en las importaciones y exportaciones en el PIB de México. En las exportaciones, en los servicios existe un porcentaje de crecimiento de 19\%, 25\%, 30\% y 38\%, respectivamente. Asimismo, existe un incremento en las importaciones de los servicios en el PIB de México, con un porcentaje de crecimiento significativo de 20\%, 27\%, 31\% y $40 \%$ en los mismos años. Esto representa que los servicios tienen un impacto porcentual alto en la economía y que México, tiene la necesidad de crear e innovar nuevos productos, debido a que las importaciones de servicios superan a las exportaciones de los mismos (Reporte del Banco Mundial, 2017).
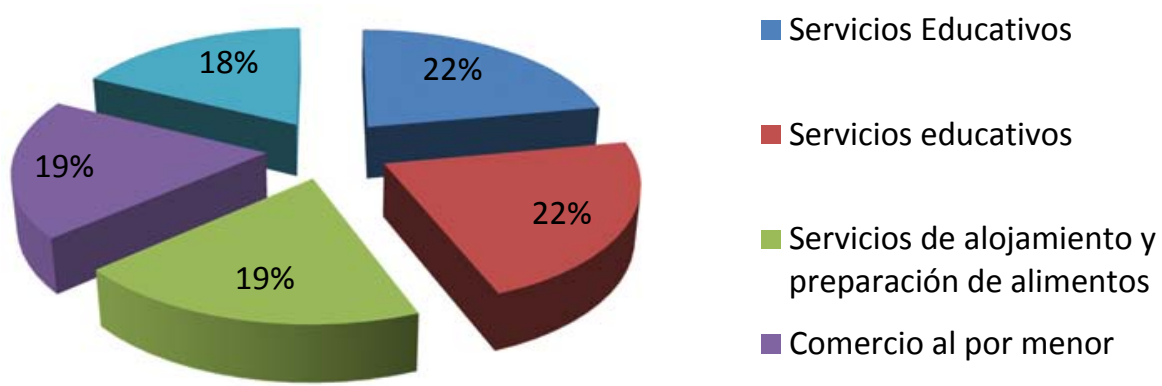

Figura 1. Mujeres involucradas en la producción de servicios.

Fuente: Elaboración propia con los datos del Censo Económico (2014) del INEGI.

Asimismo, se puede notar que incluso, desde la importancia de la actividad de los servicios, las mujeres tienen un alto grado de ocupación en este sector en el país. Según la figura 1, se presenta una relación del porcentaje de mujeres involucradas en la producción de servicios y es significativamente alta en su ocupación. 
EVALUACIÓN DE SERVICIO EN LAS EMPRESAS DE LA RUTA DEL VINO EN EL ESTADO DE GUANAJUATO.

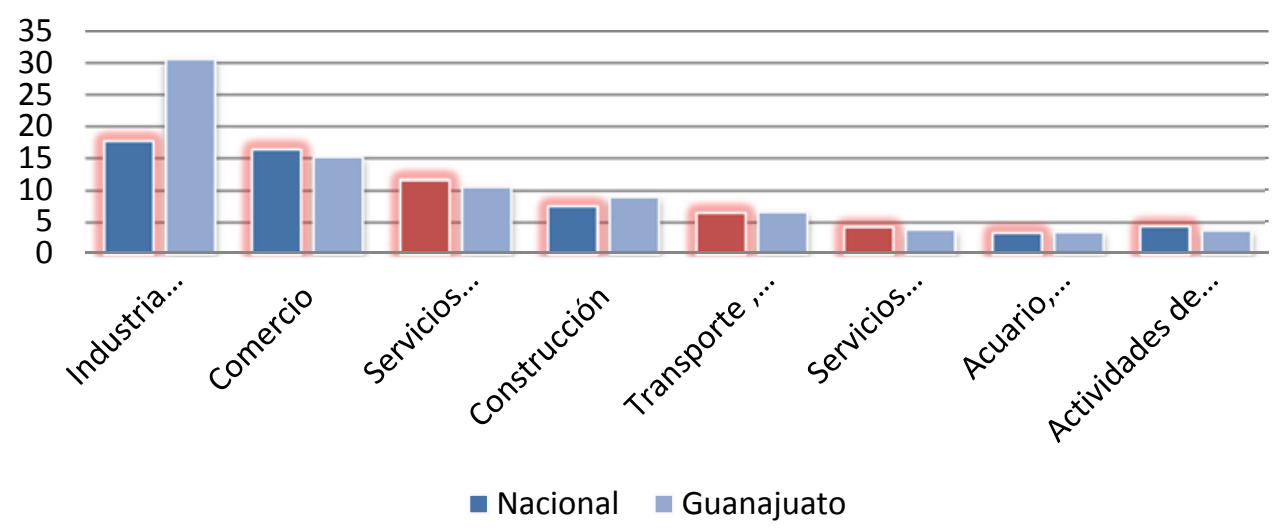

Figura 2. Porcentaje de participación de los sectores económicos de Guanajuato y a nivel nacional.

Fuente: Elaboración propia con los datos del Censo Económico del (2014) de INEGI.

En el estado de Guanajuato, el efecto de los servicios es importante, debido a que ha generado derrama económica; además, los datos del estado de Guanajuato son muy interesantes, porque refieren ocho sectores de alto impacto, dentro de los cuales varios pertenecen a servicios (ver figura 2). Se considera que las industrias que más impactan en el estado de Guanajuato son las de manufactura, toda vez que subió hasta un $30.6 \%$ en el 2014, lo cual hace un crecimiento promedio anual de $3.6 \%$ en este año. Por ello, los sectores que crecieron tienen que ver muchos con servicios, como son: transporte, correo y almacenaje, que aumentó un 3.9\%. Interesante el caso de los servicios educativos, que también repuntaron como parte del PIB, así como servicios inmobiliarios, el cual tiene una alza más grande que el resto del país, por lo menos al 2014, lo cual habla de un crecimiento interesante en diferentes sectores de la población (INEGI, 2014).

\section{MARCO TEÓRICO}

En términos conceptuales, según Duque (2005) "servicio es entonces entendido como el trabajo, la actividad y/o los beneficios que producen satisfacción a un consumidor" (p. 80). El servicio es un acto que tiene características básicas como el ser efímero, además de ser transitorio y perecedero. En general, no se pueden registrar en un inventario, aunque se puede generar la evaluación de las impresiones de manera descriptiva de la recepción del servicio. Las instalaciones, el equipo y la mano de obra, son parte de la producción del servicio y no el producto en sí mismo. En el caso de los servicios, si no hay demanda, el recurso se desperdicia y la empresa pierde el valor de estos activos, dado que está en la creación del servicio mismo. 
EVALUACIÓN DE SERVICIO EN LAS EMPRESAS DE LA RUTA DEL VINO EN EL ESTADO DE GUANAJUATO.

Según el análisis, si la demanda excede la capacidad del servicio, entonces se puede prestar a que los clientes se desilusionen por ser deficiente. Es interesante y fundamental, encontrar formas de manejar los niveles de demanda, con estrategia para ajustar la capacidad según la demanda. Existe entonces una complejidad en la demanda de los productos y servicios interesantes para poder generar la producción de los mismos (Lovelock y Wirtz, 2009).

Duque (2005) divide la evaluación de la calidad en el servicio en dos escuelas principales: la escuela Norteamericana y la escuela Nórdica. La escuela Norteamericana está representada por Parasuraman, Zeithaml y Berry, la escuela Nórdica está representada por Grönroos y es conocida como la escuela de la imagen. Este modelo habla de la relación entre la calidad y la imagen corporativa. Se refiere a que la calidad percibida es una relación entre la calidad técnica y la funcional, ambas se relacionan con la imagen corporativa. La imagen es un elemento para medir la calidad, entonces el cliente evalúa el resultado del servicio, la forma de recibirlo y su imagen corporativa; además, se estudia por separado el servicio recibido y la percepción del mismo. Por otro lado, la escuela Americana es el modelo propuesto por Parasuraman, Zeithaml y Berry $(1985,1988)$. Esta escuela creó una medición basada en dimensiones observables, que al inicio fueron 10 y luego se hicieron cinco en una segunda revisión, haciendo mención de la evaluación separada de las expectativas y las percepciones de los clientes.

Según la literatura revisada y dado que el área académica utiliza más los métodos analizados en la percepción del servicio, el modelo de la escuela de Babson College, es una derivación de los modelos anteriores, de la escuela Americana y de la escuela Nórdica (ver figura 3).

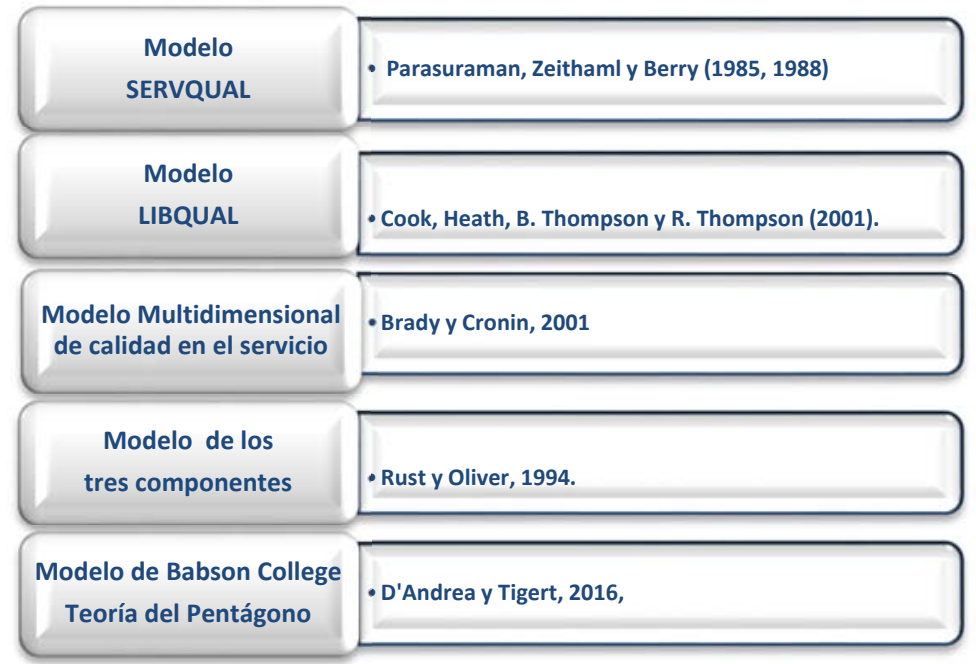

Figura 3. Evolución de los modelos de calidad para llegar al modelo de Babson College llamado la Teoría del Pentágono.

Fuente: Elaboración propia. 
EVALUACIÓN DE SERVICIO EN LAS EMPRESAS DE LA RUTA DEL VINO EN EL ESTADO DE GUANAJUATO.

A continuación, se revisan los modelos mencionados en la figura 3; el modelo SERVQUAL que proponen Parasuraman, Zeithaml y Berry (1985 y 1988), está compuesto de cinco dimensiones; la primera propuesta salió en 1985 con 10 dimensiones, pero se hizo una revisión y actualmente hay cinco elementos o dimensiones de trabajo, como son: fiabilidad, seguridad, elementos tangibles, capacidad de respuesta y empatía. El modelo de SERVQUAL, se ha usado en muchos sectores en Estados Unidos y a nivel mundial, probablemente es el más conocido desde hace muchos años en la parte académica y en la parte de las industrias. Según Ríos y Santomá (2008) citados por Torres y Vásquez (2015), este modelo permite conocer la satisfacción del usuario y determina sus brechas entre las expectativas creadas y lo que se recibe del servicio, además de medir el ámbito del sector donde se da el servicio (ver figura 4).
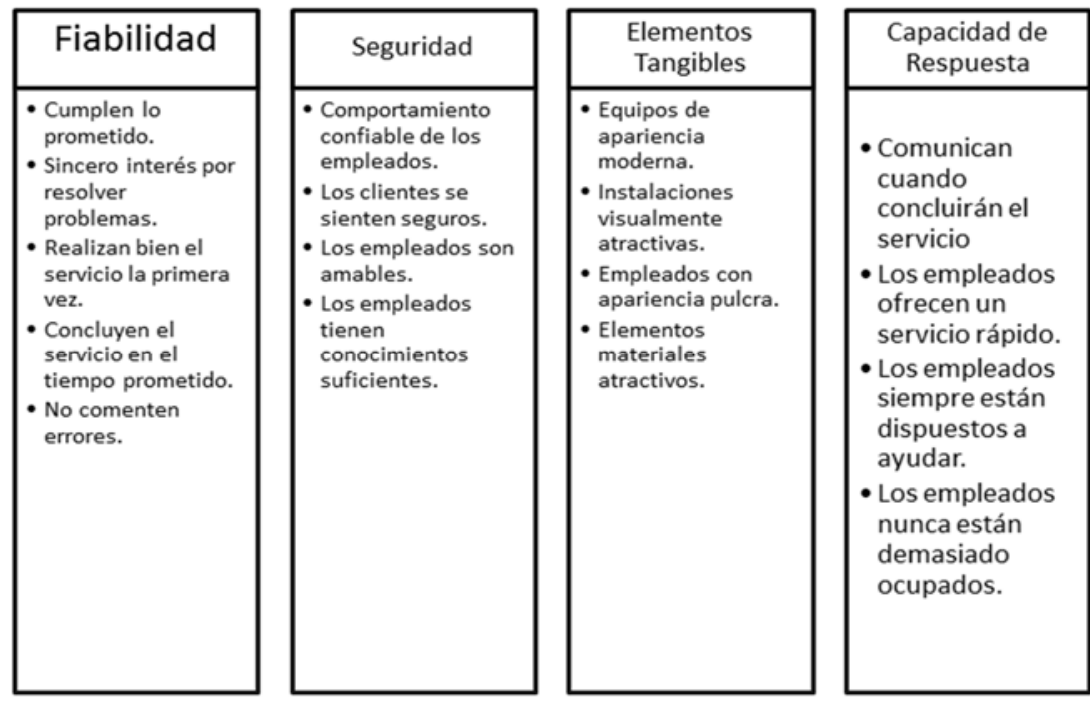

\begin{tabular}{|l|}
\hline \multicolumn{1}{|c|}{ Empatía } \\
\hline - Ofrecen \\
atención \\
individualizada. \\
- Horarios de \\
trabajo \\
convenientes \\
para los demás. \\
- Tiene \\
empleados que \\
ofrecen \\
atención \\
personalizada. \\
- Se preocupan \\
por los clientes. \\
- Comprenden las \\
necesidades de \\
los clientes. \\
\end{tabular}

Figura 4. Dimensiones e ítems del modelo de Calidad SERVQUAL, expectativas-realidad.

Fuente: Elaboración propia con información de Torres y Vásquez (2011).

Como se observa en la figura 3, en la literatura revisada existe otro modelo que se llama LIBQUAL y que se deriva de SERVQUAL; sin embargo, este nuevo modelo genera una evolución del original, conocido como SERVQUAL. Este modelo se usó para medir dimensiones en los servicios de las librerías en Estados Unidos, el cual se adapta a ciertos puntos de servicio que deben de medir varios factores, divididos en cuatro dimensiones: valor del servicio, la organización de los espacios como dimensión tangible del servicio, el acceso a la información y control de personal (ver figura 5). 

GUANAJUATO.

\section{Modelo de calidad de servicio LibQUAL}

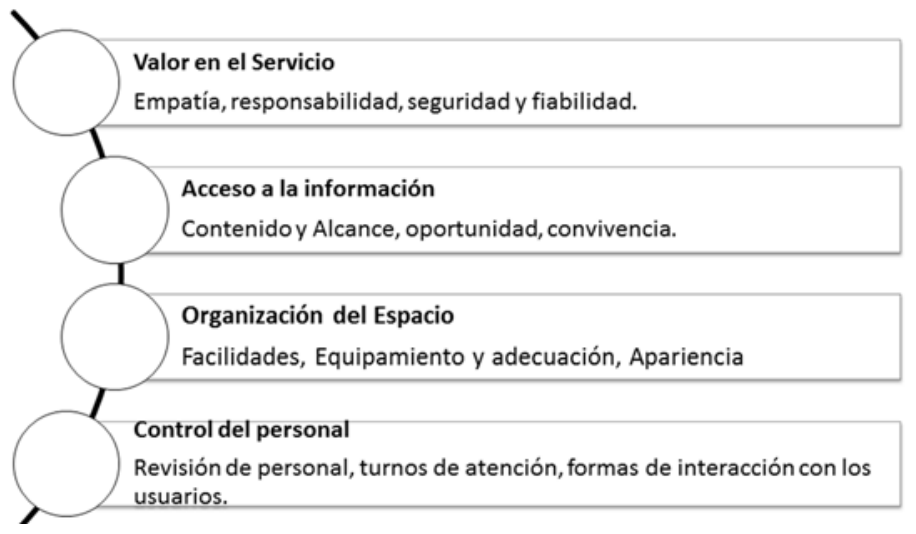

Figura 5. Modelo de Calidad de Servicio LIBQUAL.

Fuente: Elaboración propia con información de Torres y Vásquez (2011).

En relación a la línea del tiempo presentada en la figura 3, se presenta como tercer modelo, la teoría del Modelo Jerárquico Multidimensional de Brady y Cronin (2001). El servicio en este modelo es un elemento importante que considera desde hace varios años la medición y la calidad, este modelo habla que las percepciones de los usuarios son de múltiples niveles y que al final se combinan con subniveles para llegar a su percepción global. Por medio de la investigación de tipo cualitativa y de manera empírica, se demuestra que la calidad del servicio es una estructura que se define por las dimensiones y por las subdimensiones estudiadas. Este modelo de Brady y Cronin (2001) propone que se identifique: la calidad de la interacción, el ambiente físico y la calidad de los resultados (ver figura 6).

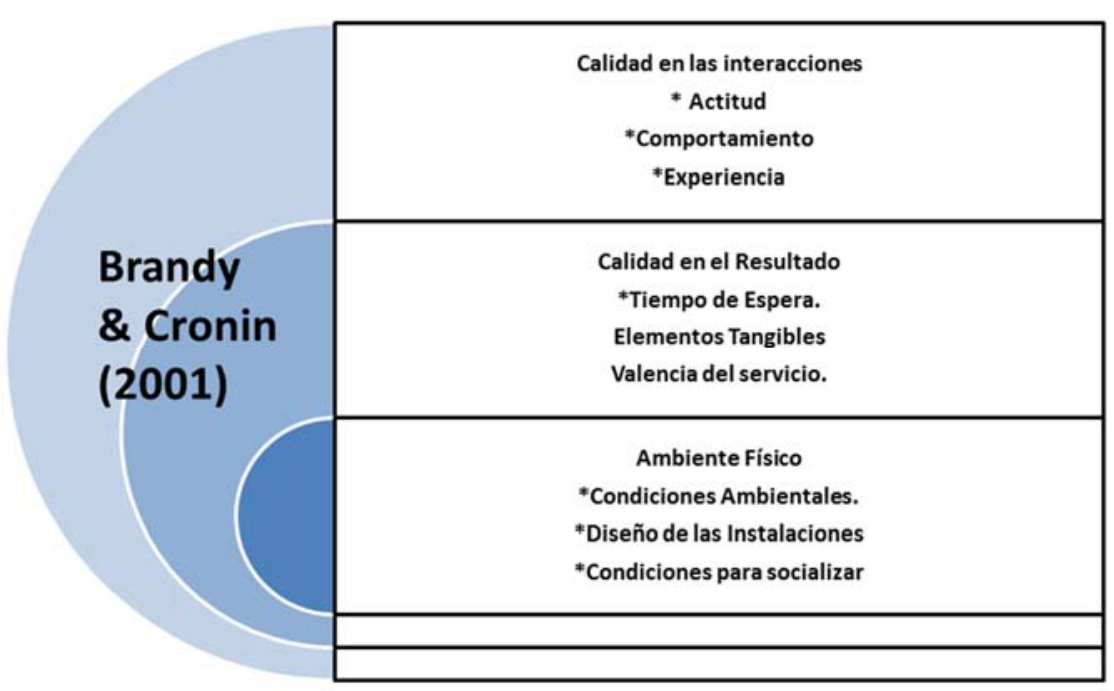

Figura 6. Modelo multidimensional de la calidad en el servicio. Fuente: Elaboración propia con información de Torres y Vásquez (2011). 
EVALUACIÓN DE SERVICIO EN LAS EMPRESAS DE LA RUTA DEL VINO EN EL ESTADO DE GUANAJUATO.

El siguiente modelo presentado en la línea del tiempo (ver figura 3), es conocido como modelo de los Tres Elementos. Según Duque (2005), para 1994 los autores Rust y Oliver, presentaron un concepto en lo planteado por la escuela Nórdica de Grönroos, este modelo propone tres elementos fundamentales: el servicio y sus características; el proceso de envío del servicio o entrega del mismo y el ambiente de la entrega. En esta ocasión, se marca un aspecto antes no considerado el ambiente de la entrega del servicio (ver figura 7).

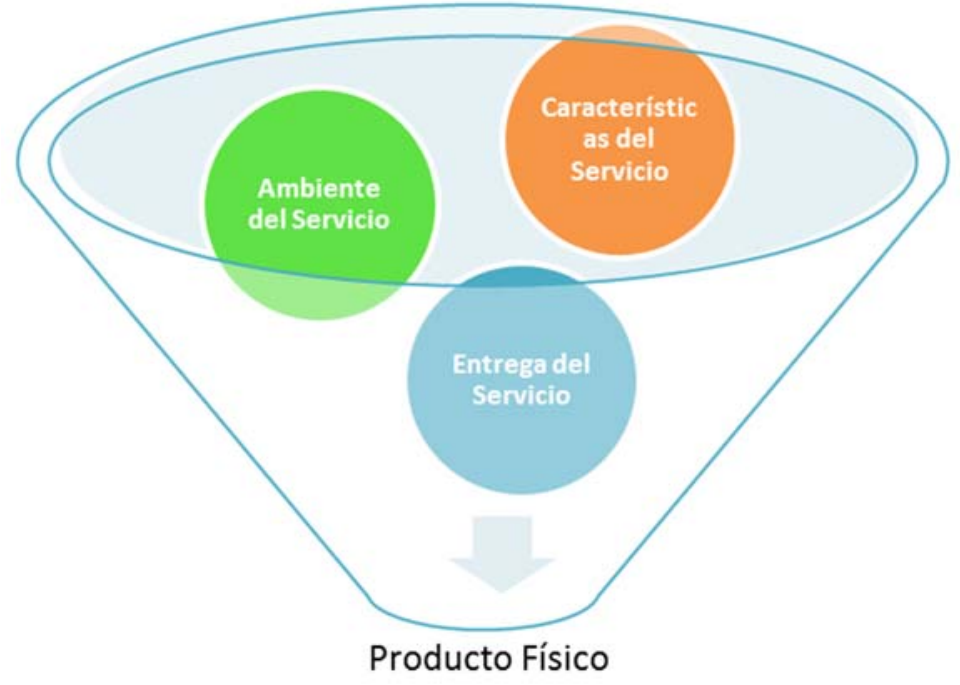

Figura 7. Modelo de los Tres Elementos.

Fuente: Elaboración propia con información de Duque (2005).

Finalmente, se presenta el modelo de Babson College, elaborado por D'Andrea y Tigert en 2005 y revisado en el (2016), actualmente establece ocho dimensiones, de las cuales cinco replantean el modelo de las 4 ps en el punto de venta. Las cinco actividades observables son: 1) el lugar, 2) el producto, 3) el valor, 4) el personal y 5) las comunicaciones. En este caso, se dirigen a los minoristas y tratan de evaluar los factores que generan tráfico en el punto de venta, se desglosan en indicadores que muestran la ubicación, el producto, personal y las comunicaciones con las que se trabajan. Todo se trabaja para reforzar "el valor ofrecido influye en el margen que los minoristas son capaces de alcanzar" (D'Andrea y Tigert, 2016, p. 200).

Existe una critica en este modelo a los otros modelos que solo miden variables como el precio y que no toman la sensibilidad del usuario para evaluar; sin embargo, este modelo se presenta confiable para la investigación ya que analiza dimensiones que otros elementos no consideran como los el punto de venta, la comunicación, las tiendas y el valor de los productos tangibles en la producción de los servicios. 
EVALUACIÓN DE SERVICIO EN LAS EMPRESAS DE LA RUTA DEL VINO EN EL ESTADO DE GUANAJUATO.

Las variables externas de este modelo mencionan cinco actividades observables, como son: 1 ) lugar, 2) producto, 3) valor, 4) personal y, 5) comunicaciones (ver figura 8); estas dimensiones son muy interesantes, porque son partes básicas de la producción del servicio al tiempo que evalúan la imagen y la producción del servicio, además incluye variables de medicion internas que por su naturaleza no fueron observadas. Ahora bien, las variables internas del modelo, son: los sistemas de compra y venta usados, la planeación de la logística y el tipo de proveedores que tiene la tienda; estos factores no pueden ser observados, debido a que dependen de la gestión interna. Como se hizo una observación no participante no se consideraron para esta investigación, pero si se considerarían para futuras descripciones e investigaciones del sector.

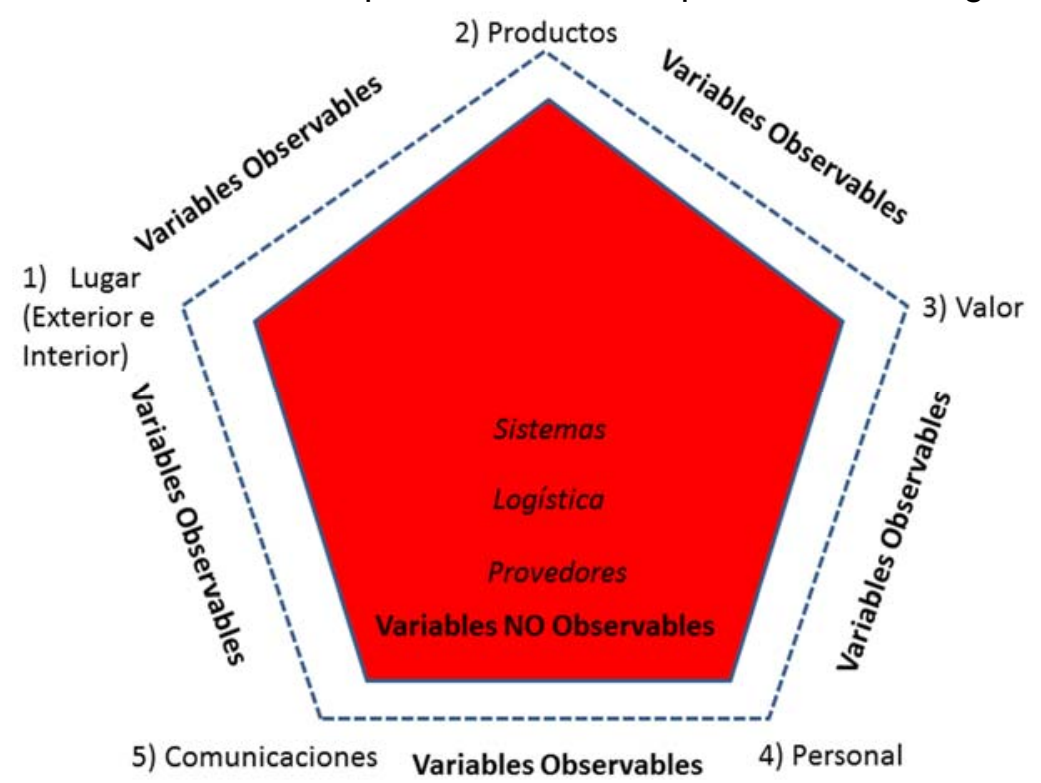

Figura 8. Modelo de Basson College.

Fuente: Elaboración propia con información de D’Andrea y Tigert (2005 y 2016).

La elección de este modelo fue clara, porque es el único que expresamente evalúa el punto de venta como un lugar de valor y los productos que se compran, el surtido que se tiene en la tienda y la producción del servicio que está en el personal y las comunicaciones del mismo. Algo muy relevante de este modelo es, que evalúa el valor de la marca como una dimensión clara; por esta razón, el modelo sirve para medir como se está compitiendo a nivel local, regional y nacional, con el servicio en el punto de venta en el caso de los viñedos estudiados.

\section{CASO DE ESTUDIO}

Según la historia en México, desde la conquista se ha producido vino; sin embargo, la historia confirma que la corona española prohibió la producción del vino. Los primeros cultivos registrados fueron, en: Puebla, Querétaro, Aguascalientes, Coahuila, California y Sonora. 
EVALUACIÓN DE SERVICIO EN LAS EMPRESAS DE LA RUTA DEL VINO EN EL ESTADO DE GUANAJUATO.

Después de largo tiempo, se retomó el proceso de fabricación del vino por cuestiones de falta de conocimiento y equipo para la producción.

El vino en Guanajuato, es una nueva industria que se ofrece como servicios de tipo enoturísticos, evalúa no sólo sus productos vitivinícolas sino las experiencias generadas en 30 viñedos que se dedican la producción, comercialización o producción de servicios turísticos en el estado de Guanajuato. Según Plano Informativo (2017), los datos de la Secretaría de Turismo de Guanajuato, comentan que existen 350 hectáreas cultivadas para la producción de la vid que elabora vino en el estado de Guanajuato. Además, ya se cuenta con aproximadamente 24 variedades de uva y 30 viñedos en operación y se producen más de 100 mil botellas y más de 23 etiquetas. Las regiones más reconocidas son: Sonora y Baja California, siendo las principales regiones productoras de uva; sin embargo, Guanajuato está entre los ocho estados de la República Mexicana que se dedican a la actividad vitivinícola y que ya tienen una derrama económica interesante en este sector.

El caso de estudio presentado es relevante para la industria vitivinícola del estado de Guanajuato, toda vez que da la oportunidad de generar instrumentos de medición para evaluar cómo se está haciendo la producción del servicio en este caso de tipo experiencia vitivinícola. Según González (2016) las cifras del tamaño de la industria vitivinícola son interesantes, donde existe un aumento del consumo del vino y existe una mejora en la calidad del vino. La industria está compuesta del vino mexicano, además de los viñedos que participan las casas productoras. Cabe destacar, que en México ya se tienen vinos nacionales premiados en concursos internacionales, específicamente en Guanajuato, donde fueron presentados en lugares importantes, como: la Ciudad del Vino, museo de experiencias vitivinícolas, ubicado en la Ciudad de Burdeos, en el país de Francia.

Asimismo, existe una promesa del consumo por la mejora de los productos vitivinícolas actuales. En 1995 surge el viñedo que es una de las empresas elegidas para este estudio. Por datos de confidencialidad no se menciona su nombre, pero es una empresa que tiene esfuerzos notables en su producción vitivinícola. Las primeras vides sembradas fueron: Cabernet Franc, Cabernet Sauvignon, Merlot, Syrah y Semillon, donde su vinificación fue en el 2005 y llegó al mercado hasta el 2008 con su vino del nombre del viñedo. El origen de esta cava es interesante, porque se revive el nombre de Dolores Hidalgo, en el Municipio de Guanajuato. El viñedo en cuestión ha ganado premios internacionales como "Premio Guanajuato a la 
EVALUACIÓN DE SERVICIO EN LAS EMPRESAS DE LA RUTA DEL VINO EN EL ESTADO DE GUANAJUATO.

competitividad Turística" como Mejor Producto (2014), Medalla de plata en la $1^{\text {a }}$ Bienal de Arquitectura por sus instalaciones. De la misma manera, la bodega con sus productos han ganado reconocimientos diversos, como: Concurso Mundial de Bruselas 2016, donde obtuvo la medalla de oro y en el 2014 , el viñedo ganó la medalla de plata en este salón, con dos productos originales creados en Dolores Hidalgo, en Guanajuato (González, 2016).

El otro caso de estudio está ubicado en la comunidad Sangre de Cristo en el municipio de Guanajuato, este viñedo es considerado el más elevado de México, ya que está a dos mil 400 metros de altura. Actualmente, el viñedo tiene buena actividad turística, donde ha llevado a cabo conciertos, teatro de calle, shows y diversas catas de vino; además, atiende a diferentes empresas de talla internacional, que utilizan sus instalaciones para realizar eventos, así como servicio de hotel, restaurante y sede de eventos particulares y oficiales en el estado de Guanajuato (Oropeza, 2015).

\section{MATERIAL Y MÉTODO}

La presente investigación es mixta, descriptiva y explicativa. Para la obtención y recopilación de información se diseñó un instrumento de medición y percepciones a través de técnicas como: la observación y la bitácora, en las cuales se analizó la información a través de un método comparativo. El estudio se aplicó con la técnica de cliente misterioso, para evitar sesgo en el comportamiento de los actores. Los factores cualitativos que se analizan son las dimensiones observadas por el modelo de D'Andrea, Ring y Tigert ( 2016). Un cliente misterioso es una técnica de observación donde se miden algunos indicadores, en realidad es una observación no participante estructurada. De acuerdo a Rábago (2011), la forma de observar y el sentido que se le da a la observación es importante, ya que se desarrollan los elementos a través de las diferentes técnicas y métodos. El enfoque derivado de la antropología hace que en las descripciones se necesite de un registro de observación. La observación consiste esencialmente en una descripción de los acontecimientos que se dan en la vida de los sujetos así como en las estructuras donde se interpreta y se le da significado a su cultura (Rábago, 2011).

En el instrumento de medición propuesto se utilizó la escala de Likert, para medir las actitudes, lo cual es muy útil, ya que representa una escala de tipo ordinal fácil de medir, donde se seleccionaron ítems específicos para su evaluación. El objeto de estudio fueron dos empresas competidoras en la ruta del vino en Guanajuato, las cuales fueron: 
EVALUACIÓN DE SERVICIO EN LAS EMPRESAS DE LA RUTA DEL VINO EN EL ESTADO DE GUANAJUATO.

Empresa 1. Viñedo en el municipio de Dolores Hidalgo en el estado de Guanajuato con premios internacionales y uno de los más conocidos a nivel México. Se omite su nombre por confidencialidad de la empresa.

Empresa 2. Viñedo en la comunidad de Sangre de Cristo, municipio de Guanajuato en el estado de Guanajuato, con presencia internacional por su realización de eventos para diferentes empresas internacionales en el municipio de Silao y de León en Guanajuato. Se omite su nombre por confidencialidad de la empresa.

La investigación fue transversal, a través de un instrumento de medición

Las dimensiones utilizadas fueron:
a. lugar (exterior, interior).
b. Exhibición de productos y servicios.
c. Personal.
d. Valor.
e. Comunicación.

Se generaron 55 ítems divididos en las diferentes dimensiones y se adaptaron a la naturaleza de las dimensiones estudiadas. La aplicación del instrumento la hicieron cuatro evaluadores por viñedo y fue creado con base en la experiencia de la evaluación del punto de venta por expertos, que previamente habían aplicado en otros sectores.

\section{RESULTADOS}

A continuación, se presentan los resultados de la investigación, basado en una calificación asignada con base a una evaluación. La evaluación utiliza una escala de Likert donde 1 es la calificación mínima y 5 la máxima. 
EVALUACIÓN DE SERVICIO EN LAS EMPRESAS DE LA RUTA DEL VINO EN EL ESTADO DE GUANAJUATO.

Tabla 3

Dimensión: Punto de venta (exterior)

\begin{tabular}{lcccc}
\hline & Evaluación Exterior & \\
\hline Aspecto evaluado: Exterior del lugar & Viñedo 1 & Viñedo 2 & Observaciones \\
\hline Fachada y rótulos identificación. & 3 & 2.67 & Es un área de oportunidad \\
Exteriores limpios con vista panorámica. & 4.33 & 3.67 & \\
$\begin{array}{l}\text { Fachada evocativa al tema. } \\
\text { Relacionada a la naturaleza del negocio. }\end{array}$ & 4.33 & 3.67 & \\
Entrada Monumental. & 3.33 & 3.67 & Es un área de oportunidad
\end{tabular}

Nota: Las ponderaciones presentadas son el resultado de 4 observaciones hechas con la técnica de cliente misterioso en los viñedos en cuestión.

Fuente: Elaboración propia.

Existe una observación interesante en el cuidado de la señalización y los muebles en general, el concepto es atractivo, pero se pierden las personas en encontrar los lugares básicos como los servicios; sin embargo, los tipos de construcción de los viñedos son agradables y están bien cuidados, lo que hace que tengan buena puntuación.

Tabla 4

Dimensión: Punto de venta (interior)

\begin{tabular}{|c|c|c|c|}
\hline \multicolumn{4}{|c|}{ Evaluación interior } \\
\hline $\begin{array}{l}\text { Aspecto evaluado: Interior del } \\
\text { lugar }\end{array}$ & Viñedo 1 & Viñedo 2 & Observaciones \\
\hline Caminos, flores, cuidado ambiental. & 4.33 & 4 & \\
\hline Accesibilidad para discapacitados. & 3 & 1.67 & Es un área de oportunidad \\
\hline Áreas Verdes y Escalas panorámicas. & 5 & 4.67 & Punto fuerte \\
\hline Lugares con transición de actividades. & 4.67 & 3.67 & \\
\hline Ciclo vías o caminos. & 2.67 & 3.67 & Es un área de oportunidad \\
\hline Amenidades, área social común. & 3.67 & 4.33 & \\
\hline Equipamiento del área social. & 3.67 & 4.33 & \\
\hline
\end{tabular}

Nota: Las ponderaciones presentadas son el resultado de 4 observaciones hechas con la técnica de cliente misterioso en los viñedos en cuestión.

Fuente: Elaboración propia. 
EVALUACIÓN DE SERVICIO EN LAS EMPRESAS DE LA RUTA DEL VINO EN EL ESTADO DE GUANAJUATO.

En cuanto a las amenidades, se encontraron lugares interesantes, pero hacen falta caminos para hacer recorridos a pie o en bicicleta más marcados. Las vistas panorámicas son muy buenas en ambos casos, pero la accesibilidad a discapacitados sobre todo en un viñedo deja mucho que desear.

Tabla 5

Dimensión 2: Productos

\begin{tabular}{|c|c|c|c|}
\hline \multicolumn{4}{|c|}{ Productos y exhibición } \\
\hline Aspecto Evaluado: Productos y Exhibición & $\begin{array}{l}\text { Viñedo } \\
1\end{array}$ & $\begin{array}{c}\text { Viñedo } \\
2\end{array}$ & Observaciones \\
\hline División de productos en zonas. & 2.33 & 4 & Es un área de oportunidad \\
\hline Productos agrupados por conceptos. & 2.33 & 4 & Es un área de oportunidad \\
\hline $\begin{array}{l}\text { Exhibición del producto de temporada en } \\
\text { aparadores exteriores. }\end{array}$ & 2 & 3.67 & Es un área de oportunidad \\
\hline $\begin{array}{l}\text { Tener } 2 \text { ó más modelos de la misma línea en } \\
\text { exhibición con los colores más llamativos. }\end{array}$ & 2.33 & 3.33 & Es un área de oportunidad \\
\hline $\begin{array}{l}\text { Agrupar en un solo mueble el producto con oferta o } \\
\text { descuento con su porta precio. }\end{array}$ & 2 & 3.33 & Es un área de oportunidad \\
\hline $\begin{array}{l}\text { Se exhibe al entrar a la tienda lo más nuevo, } \\
\text { promociones o descuentos importantes. }\end{array}$ & 2 & 2.67 & Es un área de oportunidad \\
\hline $\begin{array}{l}\text { El producto debe estar en excelentes condiciones, } \\
\text { limpio, encintado. }\end{array}$ & 3 & 4 & \\
\hline $\begin{array}{l}\text { Los modelos en existencia y los que se muestran en } \\
\text { la publicidad o en los sitios web son los mismos. }\end{array}$ & 2.67 & 3 & Es un área de oportunidad \\
\hline
\end{tabular}

Nota: Las ponderaciones presentadas son el resultado de 4 observaciones hechas con la técnica de cliente misterioso en los viñedos en cuestión.

Fuente: Elaboración propia.

La exhibición de productos en general es muy mala. No se tienen productos con exhibidores, botellas señaladas para compra venta. Si se hace la invitación y se ofrece el producto pero la comercialización da la impresión de que no es la esencia del lugar y en ambos casos se cuenta con vino propio lo que hace interesante ver que si se ofreciera o fuera más sencillo de identificar podría haber más venta. En el caso del viñedo 1, ya se tiene directo en el lugar una venta del $20 \%$ de la producción lo cual indica el servicio si da resultado, pero realmente los productos si se muestran, pero no son la causa principal del servicio. 
EVALUACIÓN DE SERVICIO EN LAS EMPRESAS DE LA RUTA DEL VINO EN EL ESTADO DE GUANAJUATO.

Tabla 6

Dimensión 2. Productos (Recorridos como parte de los productos)

\begin{tabular}{lccc}
\hline \multicolumn{4}{c}{ Productos (Recorrido Interno ) } \\
\hline Aspecto Evaluado: Recorridos internos & Viñedo 1 & Viñedo 2 & Observaciones \\
\hline Registró con exactitud el número de participantes. & 3.67 & 4.33 & \\
Informó claramente el importe a pagar y a conocer. & 4.33 & 3.33 & \\
Entrega de información correcta. & 4 & 4.33 & Punto fuerte \\
Entrega de productos, objetos. & 4 & 4 & Punto fuerte \\
Amabilidad en el recorrido. & 4 & 4.33 & Punto fuerte \\
Agradeció amablemente la visita al lugar. & 3.33 & 4.33 & \\
En general la percepción de los productos es: & 3.67 & 4.33 & \\
La innovación de los productos es: & 3.33 & 4.33 & \\
\hline
\end{tabular}

Nota: Las ponderaciones presentadas son el resultado de 4 observaciones hechas con la técnica de cliente misterioso en los viñedos en cuestión.

Fuente: Elaboración propia.

En este caso, el servicio y la demostración del lugar fueron muy bien puntuada de medio hacia alto. Uno de los puntos fuertes es el personal que está calificado y que gusta de su trabajo. La entrega de productos, la amabilidad es reconocida pero se tienen pocos productos y realmente no se sorprende mucho a menos que sea con los servicios ofrecidos. La innovación no está mal puntuada, pero se tiene que ver el tipo de evaluador para generar esta información.

Tabla 7

Dimensión 3: Valor

\begin{tabular}{|c|c|c|c|}
\hline \multicolumn{4}{|c|}{ Valor } \\
\hline Aspecto Evaluado: Valor & Viñedo 1 & Viñedo 2 & Observaciones \\
\hline Existe una propuesta de valor clara. & 4.33 & 3.67 & \\
\hline Existe una ventaja diferencial clara. & 4.33 & 3.67 & \\
\hline Existe un costo relativo al valor del producto. & 3.67 & 3.33 & $\begin{array}{l}\text { Es un área de } \\
\text { oportunidad }\end{array}$ \\
\hline $\begin{array}{l}\text { Existe un precio adecuado al producto sobre la } \\
\text { competencia. }\end{array}$ & 3 & 4 & \\
\hline Los precios están identificados en el punto de venta. & 3 & 3.33 & \\
\hline $\begin{array}{l}\text { Existe algún slogan que relaciona al precio con la } \\
\text { marca. }\end{array}$ & 2.67 & 3.67 & $\begin{array}{l}\text { Es un área de } \\
\text { oportunidad }\end{array}$ \\
\hline
\end{tabular}

Nota: Las ponderaciones presentadas son el resultado de 4 observaciones hechas con la técnica de cliente misterioso en los viñedos en cuestión.

Fuente: Elaboración propia. 
EVALUACIÓN DE SERVICIO EN LAS EMPRESAS DE LA RUTA DEL VINO EN EL ESTADO DE GUANAJUATO.

Algo interesante fue la relación valor-costo que dieron los clientes, donde no quedaron satisfechos, debido a que la puntuación no fue la más alta sino de las más bajas. De igual forma, los clientes no identifican los slogans, ni tienen claro el concepto del valor de la marca, porque los viñedos no son conocidos realmente. Solamente un viñedo es el que tiene más presencia de premios internacionales, porque se habla del valor de sus marcas de vinos y hacen énfasis en su recorrido, en comparación con los otros viñedos que no son claros.

\section{Tabla 8}

Dimensión 4: Personal

\begin{tabular}{|c|c|c|c|}
\hline \multicolumn{4}{|c|}{ Personal } \\
\hline Aspecto evaluado: Personal & Viñedo 1 & Viñedo 2 & Observaciones \\
\hline Uniforme completo, limpio y alineado. & 4 & 3.33 & \\
\hline Cabello apropiado. & 4.33 & 4.33 & \\
\hline Gafette visible. & 4 & 3 & Es un área de oportunidad \\
\hline Higiene personal. & 4.33 & 4.33 & \\
\hline Respetuosos. & 4.67 & 4.33 & \\
\hline Servicial. & 4.33 & 4.67 & Punto fuerte \\
\hline Bienvenida. & 4.33 & 4.67 & Punto fuerte \\
\hline Escuchar y proponer. & 3.33 & 4 & \\
\hline Muestra del producto y manejo de objeciones. & 3 & 4.33 & \\
\hline $\begin{array}{l}\text { Recorrido agradable en la visita por las } \\
\text { instalaciones. }\end{array}$ & 4 & 4.67 & Punto fuerte \\
\hline $\begin{array}{l}\text { Servicio en caja o venta de productos en su } \\
\text { defecto. }\end{array}$ & 3.67 & 4 & \\
\hline Despedida. & 2.67 & 4.33 & Es un área de oportunidad \\
\hline $\begin{array}{l}\text { Ofrecen tres opciones de soluciones a } \\
\text { preguntas alternas. }\end{array}$ & 2.67 & 3 & \\
\hline $\begin{array}{l}\text { Revisa el producto antes de entregar al cliente * } \\
\text { si lo hubo. }\end{array}$ & 3.67 & 5 & \\
\hline $\begin{array}{l}\text { Conocen características del lugar y de los } \\
\text { productos. }\end{array}$ & 3.33 & 4.33 & \\
\hline Orden y limpieza en el lugar de trabajo. & 4 & 5 & Punto fuerte \\
\hline
\end{tabular}

Nota: Las ponderaciones presentadas son el resultado de 4 observaciones hechas con la técnica de cliente misterioso en los viñedos en cuestión.

Fuente: Elaboración propia. 
EVALUACIÓN DE SERVICIO EN LAS EMPRESAS DE LA RUTA DEL VINO EN EL ESTADO DE GUANAJUATO.

Es muy interesante reconocer que el personal en general es bueno para la atención, normalmente se tiene una recepción adecuada y se atiende de manera amable en ambos viñedos. Situaciones culturales a marcar son las despedidas que se puntuaron como baja y que los clientes esperan. Además la entrega de productos en un viñedo, si bien no tienen mucha variedad, tuvo una puntuación de 5, lo cual es sumamente bueno para el mercado.

Tabla 9

Dimensión 5: Comunicación

\section{Proceso de comunicación}

\begin{tabular}{lccc}
\hline Aspecto evaluado: Proceso de comunicación & Viñedo 1 & Viñedo 2 & Observaciones \\
\hline Existe un logotipo claro. & 2.67 & 3.33 & $\begin{array}{c}\text { Es un área de } \\
\text { oportunidad }\end{array}$ \\
El logotipo expresa concepto de producto. & 3 & 3.67 & 4.33 \\
$\begin{array}{l}\text { Imagen en la tienda adecuada al concepto. } \\
\text { Existe mención de la marca o visualización de la marca. }\end{array}$ & 3 & 4.67 & \\
$\begin{array}{l}\text { Tienen guiones de venta marcados con el nombre de la } \\
\text { marca. }\end{array}$ & 3 & 3.67 & \\
$\begin{array}{l}\text { Se genera ambiente adecuado al concepto de la marca } \\
\text { por los empleados. }\end{array}$ & 4 & 4.33 & \\
\hline
\end{tabular}

Nota: Las ponderaciones presentadas son el resultado de 4 observaciones hechas con la técnica de cliente misterioso en los viñedos en cuestión.

Fuente: Elaboración propia.

En general en la comunicación se denota que los logotipos son poco reconocidos, ya que no tiene mucha presencia de marca o logos en el lugar. Es interesante que los logotipos no se relacionan mucho con el concepto y solo en el caso de un viñedo que tiene hotel se considera que existe visualización de la marca porque el primer viñedo es una fábrica más que un restaurante. Los guiones de nombre de marca se reconocen a medias y el ambiente si refleja la marca.

\section{CONCLUSIONES}

Existen diferentes áreas de oportunidad y de trabajo de mercadotecnia en los servicios estudiados. A nivel comercial, los viñedos son reconocidos por sus recorridos y las experiencias 
EVALUACIÓN DE SERVICIO EN LAS EMPRESAS DE LA RUTA DEL VINO EN EL ESTADO DE GUANAJUATO.

enoturísticas; por esta razón, la sensación de ir al viñedo es reconocida, pero en la cuestión de evaluación del servicio al cliente en el punto de venta se tiene aspectos mejorables.

En la parte física, los viñedos necesitan más fachadas evocativas que den al punto de venta las características y que transmitan concepto de marca, así como poner atención en la señalización y en la parte de los puntos de acceso para las personas con alguna discapacidad. Además, se deben enfocar en las secciones para despertar el interés de los consumidores en los temas vitivinícolas y generar una experiencia de diversión y de consumo más amplia.

En la cuestión del personal, el trabajo está bien realizado y el personal está capacitado pero se les olvida cerrar el servicio con los detalles como despedidas, venta de producto, monitoreo de los servicios postventa. Estos elementos son los factores claves para los clientes que buscan regresar o evaluar de manera alta la experiencia del servicio vitivinícola. Las experiencias son consideradas de inicio a fin para el cliente y se debe trabajar más en esta área para mejorar la calidad de la información proporcionada al cliente por los servidores del lugar.

En cuanto a la connotación de valor, los clientes no puntúan muy bien la relación costobeneficio, lo que hace que sea interesante la relación del valor que de repente no es la mejor porque no se ve el producto o no se entiende el concepto que se está vendiendo. Es importante trabajar más en la marca o el branding, se debe generar más difusión y hacer más notorio el servicio ofrecido; es decir, se requiere de hacer más publicidad e imagen dentro del lugar, para que el cliente entienda el concepto que se vende y que se puedan ver las experiencias finales en la recomendación de la marca.

La comunicación de la marca no es muy buena y no se identifica desde el inicio. No se conocen los logotipos ni las partes de la identidad gráfica, que son el factor físico y el emocional para generar un amor a la marca; en otras palabras, no se tiene un distintivo comunicativo en colores que sea reconocido por el visitante que va al lugar. Algo importante es, que tampoco se hace referencia a un valor emocional que despierte alguna identidad con la marca, simplemente se reconocen los logos en el lugar pero requieren de más explicación o experiencia para que puedan ser queridos.

Las experiencias como los recorridos gustaron mucho; por ello, se recomienda investigar qué nuevas propuestas se podrían adjuntar a los recorridos para mejorar, lo cual sería un punto de 
EVALUACIÓN DE SERVICIO EN LAS EMPRESAS DE LA RUTA DEL VINO EN EL ESTADO DE GUANAJUATO.

partida para una nueva investigación e identificar las necesidades del cliente para su satisfacción.

En general, las puntuaciones fueron de 3 y 4, lo que indica que se tiene una percepción media del producto o servicio, aun cuando se trata de algo especializado, lo que hace pensar que la satisfacción al cliente es media y que se debe trabajar para hacer una experiencia inolvidable en cada parte del servicio, para que las personas decidan volver.

El sector vitivinícola en Guanajuato, tiene muchas áreas de oportunidad comerciales en la oferta actual de servicios; por tanto, es interesante valorar cuales son las mejores experiencias y tratar de repetirlas, para que el mercado lo valore y logre que lo servicios sean buscados. De ahí, las rutas vitivinícolas son experiencias que pueden ser novedosas, pero requieren de factores sorpresa para que el mercado evoque sensaciones diferentes y sean productos valorados. En definitiva, se presenta un reto en el área de punto de venta y de inteligencia comercial, con el propósito de detonar nuevas ideas y oportunidades de mercado dentro de los viñedos estudiados.

\section{REFERENCIAS BIBLIOGRÁFICAS}

Banco Mundial. (2017). Comercio de servicios (\% del PIB). Recuperado de https://datos.bancomundial.org/indicador/BG.GSR.NFSV.GD.ZS

Banco Mundial. (2017). Country profile: México. Recuperado de https://databank.worldbank.org/data/views/reports/reportwidget.aspx?Report_Name=Cou ntryProfile\&ld=b450fd57\&tbar=y\&dd=y\&inf=n\&zm=n\&country=MEX

Brady, M. y Cronin, J. (2001). Some new thoughts on conceptualizing perceived services quality: a hierarchical approach. Journal of Marketing, 65(3), 34-49.

Cook, C., Heath, F., Thompson, B. y Thompson, R. (2001). The search for new measures: The ARL LibQUAL + Project-- A preliminary report. Portal Libraries and the Academy, 1(1), 103-112.

Cronin J. y Taylor, S. (1992). Measuring service quality: A reexamination and extension. Journal of Marketing, 56, 55-68. 
EVALUACIÓN DE SERVICIO EN LAS EMPRESAS DE LA RUTA DEL VINO EN EL ESTADO DE GUANAJUATO.

D'Andrea, G., Ring, L. y Tigert, D. (2016). Retail Management: claves de la estrategia y la gestión minorista. Buenos Aires, Argentina: Temas.

Duque, E. (2005). Revisión del concepto de calidad del servicio y sus modelos de medición. INNOVAR. Revista de ciencias administrativas y sociales, 15(25), 64-80.

González, M. (23 de septiembre de 2016). Los otros viñedos de México: de Chihuahua a Guanajuato. Sin Embargo. Recuperado de https://www.sinembargo.mx/23-092016/3094569

INEGI. (2014). Reporte de estructura económica del estado de Guanajuato. Aguascalientes, Aguascalientes. INEGI.

Lovelock, C. y Wirtz, J. (2009). Mercadotecnia de servicios: personal, tecnología y estrategias. México: Pearson Educación.

Oropeza, P. (27 de julio de 2015). Camino D’Vinos, nuevo viñedo de Guanajuato. El Financiero. Recuperado de https://www.elfinanciero.com.mx/bajio/caminos-d-vinos-nuevo-vinedo-deguanajuato

Parasuraman, A., Zeithaml, V. y Berry, L. (1985). A conceptual model of service quality and its implications for future research. Journal of Marketing, 49(4), 41-50.

Plano Informativo. (08 de julio de 2017). Guanajuato se posiciona en producción de vino. Recuperado de https://planoinformativo.com/531743/guanajuato-se-posiciona-enproduccion-de-vino-negocios

Rábago, F. (2011). Líneas de investigación cualitativa. Guanajuato: Universidad de Guanajuato.

Rust, R. y Oliver, R. (1994). Service quality: new directions in theory and practice. California: Sage Publications.

Torres, M. y Vásquez, C. (2011). Contribución de la información en la calidad de los servicios. Enl@ce: Revista Venezolana de Información, Tecnología y Conocimiento, 8(1), 55-70.

Torres, M. y Vásquez, C. (2015). Modelos de evaluación de la calidad del servicio: caracterización y análisis. COMPENDIUM, (35), 57-73. 